\title{
The cosmic ray detector for the NICA collider
}

Marcin Bielewicz ${ }^{1,2, *}$, Martyna Grodzicka-Kobylka ${ }^{1}$, Bartosz Maksiak ${ }^{1}$, Slawomir Mianowski ${ }^{1}$, Damian Pszczel $^{1}$, Pawel Sibczynski ${ }^{1}$, Joanna Stepaniak ${ }^{1}$, Lukasz Swiderski ${ }^{1}$, Tomasz Szczesniak ${ }^{1}$, Maciej Linczuk ${ }^{3}$, Daniel Wielanek ${ }^{3}$, Adam Kisiel $^{3,2}$, Grzegorz Kasprowicz ${ }^{3}$, Krzysztof Pozniak ${ }^{3}$, Wojciech Zabołotny ${ }^{3}$, Ryszard Romaniuk ${ }^{3}$, Arkadiusz Chlopik ${ }^{1}$, Aleksandr Bancer $^{1}$, Krystian Grodzicki ${ }^{1}$, Adam Dudzinski ${ }^{1}$, Elzbieta Jaworska ${ }^{1}$, Elzbieta Strugalska-Gola ${ }^{1}$, Marcin Szuta $^{1}$, Marek J. Peryt ${ }^{3,2}$, Krystian Roslon ${ }^{3,22}$, Maciej Rybczynski ${ }^{4}$, Zbigniew Wlodarczyk ${ }^{4}$, Grzegorz Stefanek ${ }^{4}$, Pawel Kankiewicz $^{4}$, Agnieszka Synfeld-Kazuch ${ }^{1}$, and Michalina Milewicz-Zalewska ${ }^{2}$

${ }^{1}$ National Centre for Nuclear Research, ul. Andrzeja Sołtana 7, 05-400 Otwock, Świerk, Poland

${ }^{2}$ Joint Institute of Nuclear Research, Joliot-Curie, 6 Dubna, Moscow region, Russia, 141980

${ }^{3}$ Warsaw University of Technology, Pl. Politechniki 1, 00-661 Warsaw, Poland

${ }^{4}$ Jan Kochanowski University, ul. Żeromskiego 5, 25-369 Kielce, Poland

\begin{abstract}
Multi-Purpose Detector (MPD) is a main part of a new Ion Collider fAcility (NICA) located in Dubna, Russia. To increase MPD functionality, it was proposed to add an additional muon trigger system for off-beam calibration of the MPD sub-detectors and for rejection of cosmic ray background during experiments. The system could also be very useful for astrophysical observations of cosmic showers initiated by high energy primary particles. This article describes the main goals of MCORD detector and the early stage of MCORD design, based on plastic scintillators with silicon photomultiplier photodetectors (SiPM) for scintillation readout and electronic system based on MicroTCA standard.
\end{abstract}

\section{Introduction}

The new collider complex NICA (Nuclotron-based Ion Collider fAcility) with two circle accelerators (Booster and Nuclotron) is currently under construction at the Joint Institute for Nuclear Research (JINR) in Dubna, Russia [1]. At the first stage of operation the main detection system at NICA will be the Multi-Purpose Detector (MPD) [2] (Fig.1).

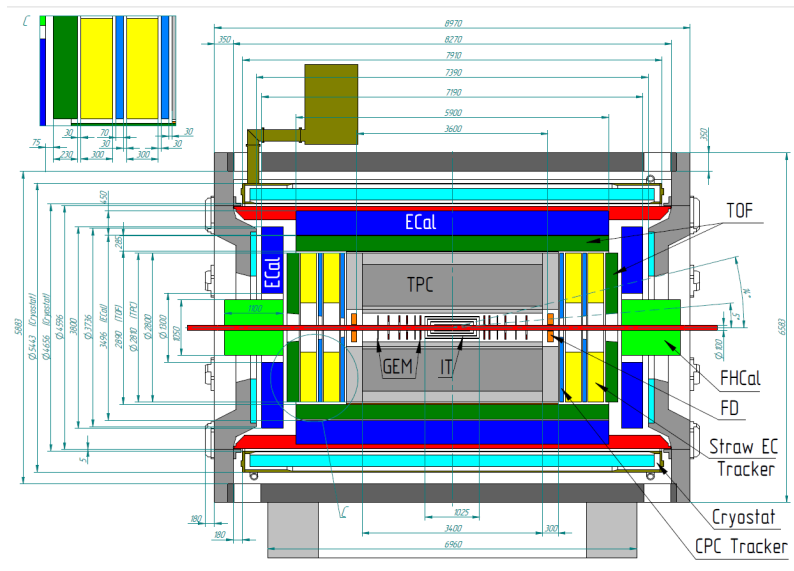

Figure 1. Cross Section of the MPD - the main NICA detection system. [2]

\section{Motivation}

The MPD was designed to identify particles emitted during ion-ion collisions. Recently, it was proposed to increase MPD functionality by installing an additional detection system surrounding the MPD, namely Muon Cosmic Ray Detector (MCORD), see Fig.2. The motivation for designing and constructing the MCORD detector is:

1. Providing trigger for testing and calibration of other sub-detector systems before completion of MPD, e.g.: Time of Flight (TOF), Electromagnetic Calorimeter (ECAL) and Time Projection Chamber (TPC).

2. Identification of muons produced as a result of ionion collisions inside the MPD.

3. Providing data for astrophysical observations (similarly to ACORDE detector in ALICE experiment at LHC [3]), e.g. deducing directionality of muon showers [4].

4. Vetoing for rare events research to reduce cosmic ray background.

\section{MCORD System Decription}

MCORD will provide information about the time, position, direction and amplitude of signal induced by charged 

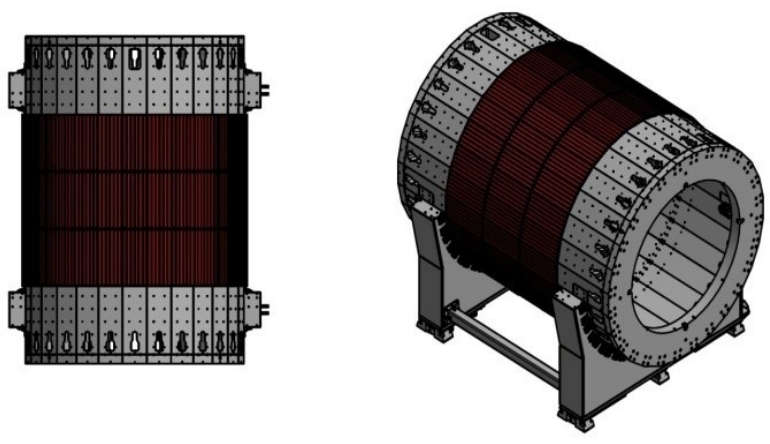

Figure 2. The MCORD modules on the surface of the MPD magnet yoke.

particles passing through the scintillators. Since the MPD is a large scale device (about 8 meters in length and 6 meters in diameter), the MCORD needs to be designed with relatively cheap materials and cost efficient assembly. It was decided to use plastic scintillator bars equipped with wavelength shifting fibers. The MPD detector comprises a large magnet around which the MCORD detector should be installed, light readout will be done by means of silicon photomultipliers (SiPMs) [5], as they are insensitive to magnetic field. Each MCORD module (Fig.3) will consist of three sections (Fig.4) and each section will comprise 8 plastic scintillators with aluminum cover. At each end of a scintillator there will be a SiPM photodetector with a dedicated power supply, a temperature compensation circuit and an amplifier. As a result, one MCORD module will comprise 48 measurement channels. The SiPMs and related electronics (Fig.5) will be embedded into scintillators aluminum cover with end-cups that will shield the scintillators from light and protect them from dust and water. The output signal from a SiPM will be amplified and shaped in an analog front end module (Fig.5) and subsequently will be sent to a MicroTCA System (MicroTCA $®$ is a modular, open standard for building high performance switched fabric computer systems in a small form factor [6]) (Fig.6). The TCA standard allows to install up to 12 AMC card (Advanced Mezzanine Card). Industry standard AMC-FMC carriers will be used for MCORD system. FMC (FPGA Mezzanine Card) is an ANSI standard that provides a standard mezzanine card form factor, connectors, and modular interface to an FPGA located on a base board (Fig.7). For more information about light production and propagation, analog and digital electronic system see $[7,8]$.

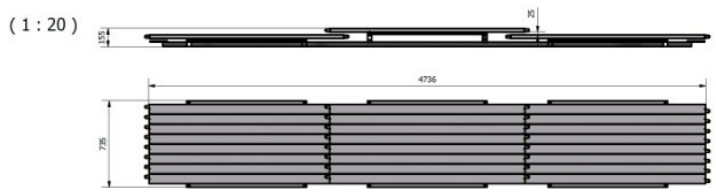

Figure 3. The MCORD modules consist of three sections, eight scintillators each.

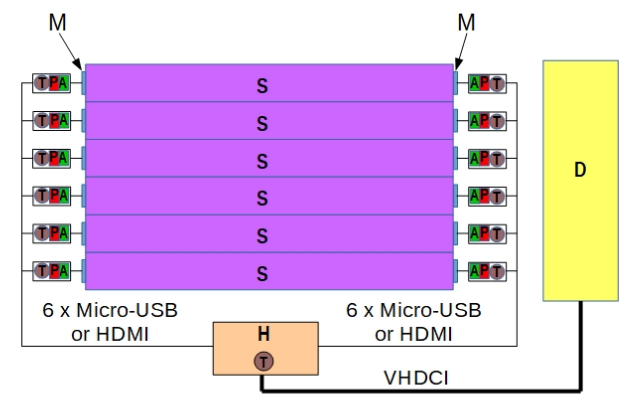

Figure 4. Conceptual design of the MCORD detector readout section. Legend: S (purple) - plastic scintillator, (blue-both ends of scintillators) - SiPMs, P (red) - power supply with temperature compensation circuit, $\mathrm{T}$ (brown) - temp. sensor, A (green)amplifier, $\mathrm{H}$ (orange) - passive HUB,D (yellow) - MicroTCA digital system, A-P-T-H - Analog Front End Module.

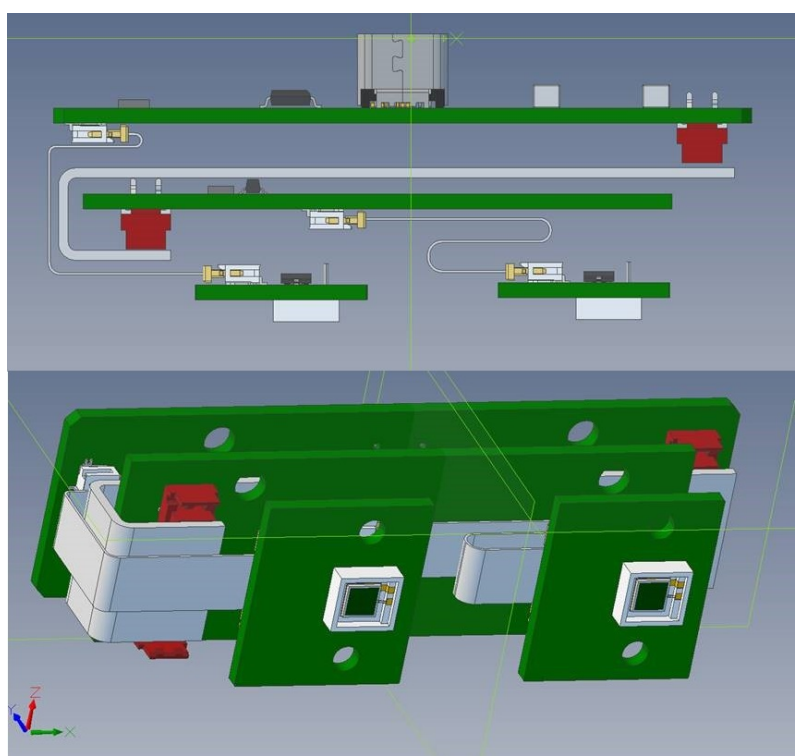

Figure 5. The separated electronic PCBs with SiPMs, amplifier and power supply.

The scintillators size will be about $1620 \times 72 \times 22 \mathrm{~mm} 3$. The time difference in light propagation through the fiber embedded into plastic will be used to find the particle interaction position along the scintillators module, whereas the width and thickness of the plastic slab will give upper limits on the muon track position in the plane perpendicular to the long detector axis. Application of fiber for light collection along the scintillating bars allows for using small SiPM photodetectors $(1.3 \times 1.3 \mathrm{~mm} 2)$.

MCORD detector modules will be arranged in a barrel shape around the shielding of the MPD detector (Fig.2). Each module will be installed on the MPD shielding surface separately (Fig.8). Signals induced by muons will be processed in the FPGA basing on coincident hits from different detector layers allowing for particle tracking. The 


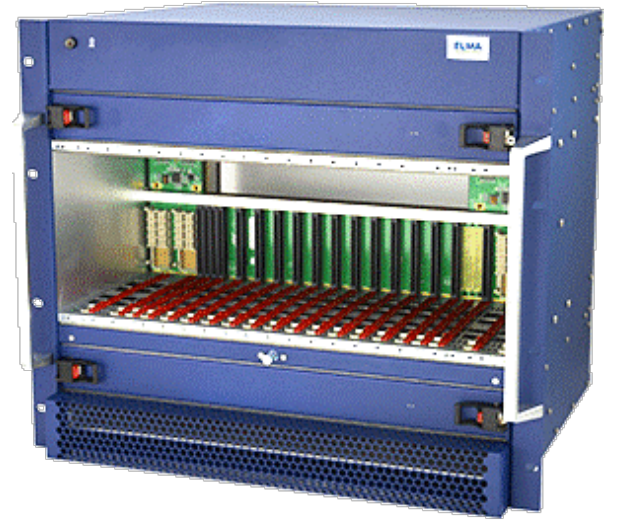

Figure 6. MicroTCA4 crate with 12 slots PICMG.
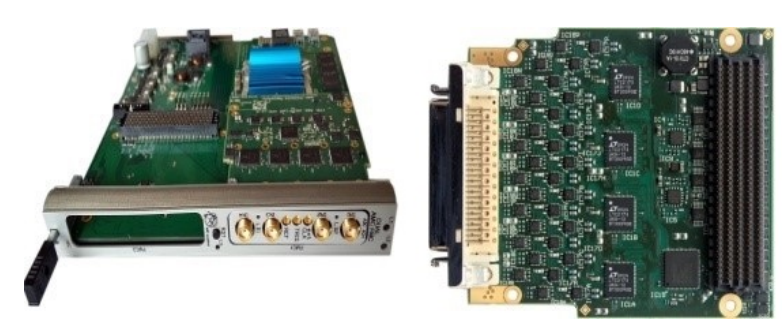

Figure 7. The separated electronic PCBs with for SiPMs, amplifier and power supply.

fast and low latency process of a muons trajectory identification will be based on the muon trajectory processor. The original concept for the Overlap Muon Track Finder can be found in [9], [10], while the CMS muon trigger system is decribed in [11].

\section{Summary}

This work is a part of the activities of a NICA-PL Consortium. The paper presents the current concept of a modular cosmic muon trigger system for the MPD detector at NICA facility. Detection system is based on low cost elements (plastic scintillators with SiPMs photodetectors). The digital analysis system will be based on the MicroTCA standard with FPGA modules. The proposed hardware can be modified upon requests to change required number of channels or spatial resolution.

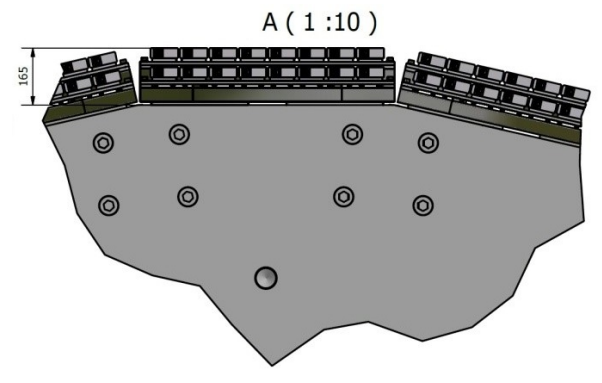

Figure 8. MCORD modules on the MPD surface.

\section{Acknowledgements}

This work is partially supported by Research Program for the research group at JINR and research centres in Poland 2019 and Polish National Science Centre (NCN) grant 2016/23/B/ST2/00692. This work is a part of Polish Consortium for NICA program (NICA-PL).

\section{References}

[1] Ref.: http://nica.jinr.ru/complex.php

[2] V. Golovatyuk, V. Kekelidze, V. Kolesnikov, O. Rogachevsky1and A. Sorin The Multi-Purpose Detector (MPD) of the collider experiment, Eur. Phys. J. A (2016) 52: 212

[3] A. Fernandez, S. Kartal, C. Pagliarone, ACORDE a Cosmic Ray Detector for ALICE, arXiv : physics/0612051 [physics.ins-det]

[4] Ref.: https://en.wikipedia.org/wiki/Air_shower_ (physics)

[5] M. Grodzicka-Kobylka, T. Szcześniak, M. Moszyński, Comparison of SensL and Hamamatsu $4 \times 4$ channel SiPM arrays in gamma spectrometry with scintillators, Nucl. Instrum. and Meth. in Phys. Res. A, Vol. 856, p.53 (2017)

[6] Ref.: https://www.picmg.org/openstandards/microtca/

[7] M. Bielewicz et al., MCORD: MPD cosmic ray detector for NICA, Proc. SPIE Vol. 10808 No. 1080847 (2018)

[8] M. Bielewicz et al., MCORD - MPD Cosmic Ray Detector a new features, EPJ Web Conf. Vol. 204 No 07016 (2019)

[9] W.M. Zabołotny et al., Implementation of the data acquisition system for the Overlap Muon Track Finder in the CMS experiment, Journal of Instrumentation, Vol. 12, p. C01050 (2017)

[10] W.M. Zabolotny, A. Byszuk, Algorithm and implementation of muon trigger and data transmission system for barrel-endcap overlap region of the CMS detector, Journal of Instrumentation, Vol. 11, p. C03004 (2016)

[11] U Bhawandeep et al., The CMS trigger system, Journal of Instrumentation, Vol. 12, pp. 1-122 (2017) 